\title{
Successful Surgical Treatment of a Patient with Synchronic Intraductal Papillary Mucinous Carcinoma and Solid Pseudopapillary Neoplasm of the Pancreas
}

\author{
Andrey G. Kriger David S. Gorin Stanislav V. Berelavichus \\ Vladimir I. Panteleev Ayrat R. Kaldarov
}

A.V. Vishnevsky Centre of Surgery, Moscow, Russian Federation

\section{Keywords}

Intraductal papillary mucinous neoplasm · Solid pseudopapillary neoplasm · Pancreas

\section{Abstract}

Intraductal papillary mucinous neoplasm (IPMN) and solid pseudopapillary neoplasm (SPN) are uncommon cystic tumors of the pancreas. The simultaneous occurrence of these 2 pancreatic neoplasms is extremely rare. We present a case of synchronous solid SPN and IPMN carcinoma in a 44-year-old female patient. An uncommon surgical treatment was performedpancreatoduodenectomy with the resection of the pancreas body, resection of the mesentericoportal trunk + distal pancreatectomy, and splenectomy. Part of the pancreatic tissue was preserved.

\section{Introduction}

Intraductal papillary mucinous neoplasm (IPMN) is a relatively rare cystic tumor of the pancreas which occurs in $0.31-4.35$ per 100,000 cases per year [1]. Solid pseudopapillary neoplasm (SPN) was described by Virginia Frantz in 1959 and is also one of the rarest tumors of the pancreas [2]. There are $<6,000$ cases of SPN described in the world literature [3]. The combination of these 2 pancreatic neoplasms is extremely rare. We present such a case of synchronous SPN and IPMN in a young patient. 

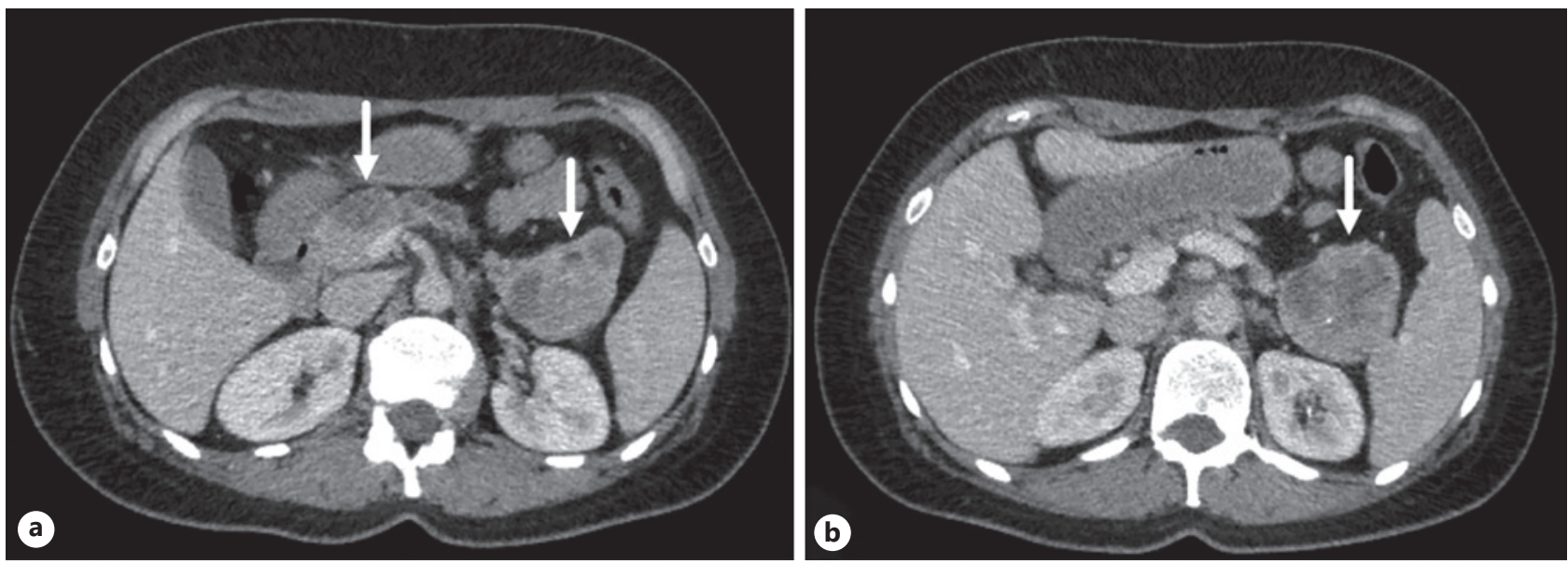

Fig. 1. Preoperative data. a CT scan, axial view, venous phase: tumors of the head and tail of the pancreas, and dilated pancreatic duct. b CT scan, axial view: tumor of the tail of the pancreas.

\section{Case Presentation}

A 44-year-old female was admitted to our center suffering from periodic pain of low intensity in the upper abdomen. At the age of 14 years, SPN was diagnosed and histologically confirmed. Surgery was not suggested to the patient and she lived with this diagnosis for 30 years. A control computed tomography (CT) in 2018 detected another tumor, located in the pancreatic head as well as the dilated pancreatic duct (maximal diameter $-6 \mathrm{~mm}$ ). Thus, the supposed diagnosis was 2 SPN located in the head and tail of the pancreas. With this diagnosis the patient came for consultation to our center in July 2019. Other medical history was unremarkable. There was no weight loss. At admission, her condition was satisfactory. The abdomen was of normal size, soft, and painless. Tumor masses were not determined by palpation. There were no abnormalities in clinical or biochemical blood tests. According to preoperative contrast-enhanced CT and MRI, the tumor in the head of the pancreas was most likely an intraductal papillary mucinous type I carcinoma with invasion into the superior mesenteric vein; the mass in the tail was a solid pseudopapillary tumor (Fig. 1).

The preoperative diagnosis was multiple primary tumors of the pancreas - intraductal papillary mucinous carcinoma (IPMC) of the head and an SPN of the tail of the pancreas. Surgical treatment was performed - pancreatoduodenectomy with resection of the pancreas body, resection of the mesentericoportal trunk, distal pancreatectomy, and splenectomy.

Intraoperatively, there were no signs of metastatic process. The tumor was detected in the neck of the pancreas with spread to the head and proximal part of the body. In the tail of the pancreas a similar tumor was identified. When the tumor in the head was mobilized it was revealed that it adheres tightly to the mesentericoportal trunk. The common hepatic duct was transected. The gland was transected at the level of the body. The pancreatic duct was $4 \mathrm{~mm}$; the parenchyma of the pancreas was atrophic. The head of the gland was partially mobilized and then resected for frozen section analysis. The pancreatoduodenal complex was then removed. On the portal vein in the confluence region there was a fragment of tissue tightly fixed to the venal walls (Fig. 2). The intraoperative frozen section revealed the presence of carcinoma cells. Then, the capsule of the tumor in the tail was separated from the splenic artery. The splenic vein was separated from the body at the level of the distal pole of the tumor. Distal resection of the pancreas with splenectomy was then performed. The remaining fragment of the pancreas was $3-4 \mathrm{~cm}$, its tissue was dense, and arterial and venous blood supply was preserved. It was decided to abstain from extirpation of this remaining part. Then, the mesentericoportal trunk was resected, and prosthetics with Ecoflon 11-mm prosthesis was performed without suturing of the splenic vein. The length of time the blood flow was absent in the portal and mesenteric veins was $25 \mathrm{~min}$. The resection was complete, and anastomoses were formed. 


\section{Tumors}
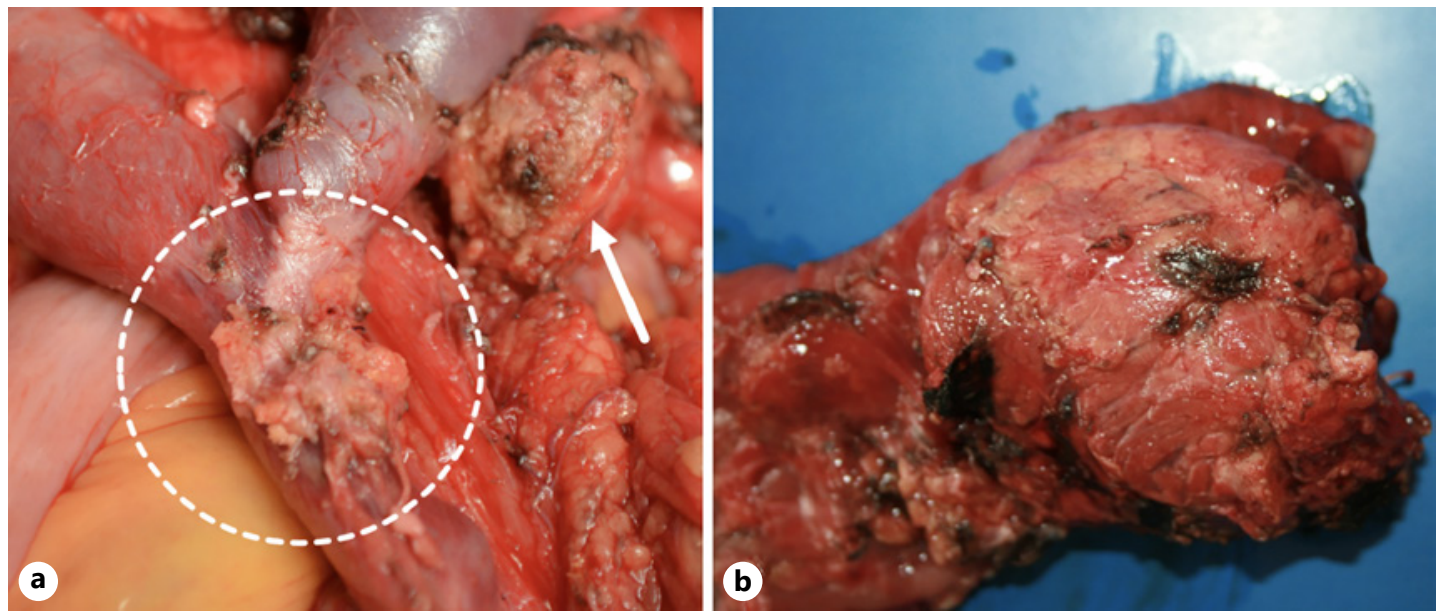

Fig. 2. Intra- and postoperative data. a Intraoperative view after removal of the pancreatoduodenal complex. Tumor growth can be seen on the mesentericoportal trunk; the arrow indicates the remaining part of the pancreatic parenchyma. b Specimen: intraductal papillary mucinous neoplasm associated with ductal adenocarcinoma.
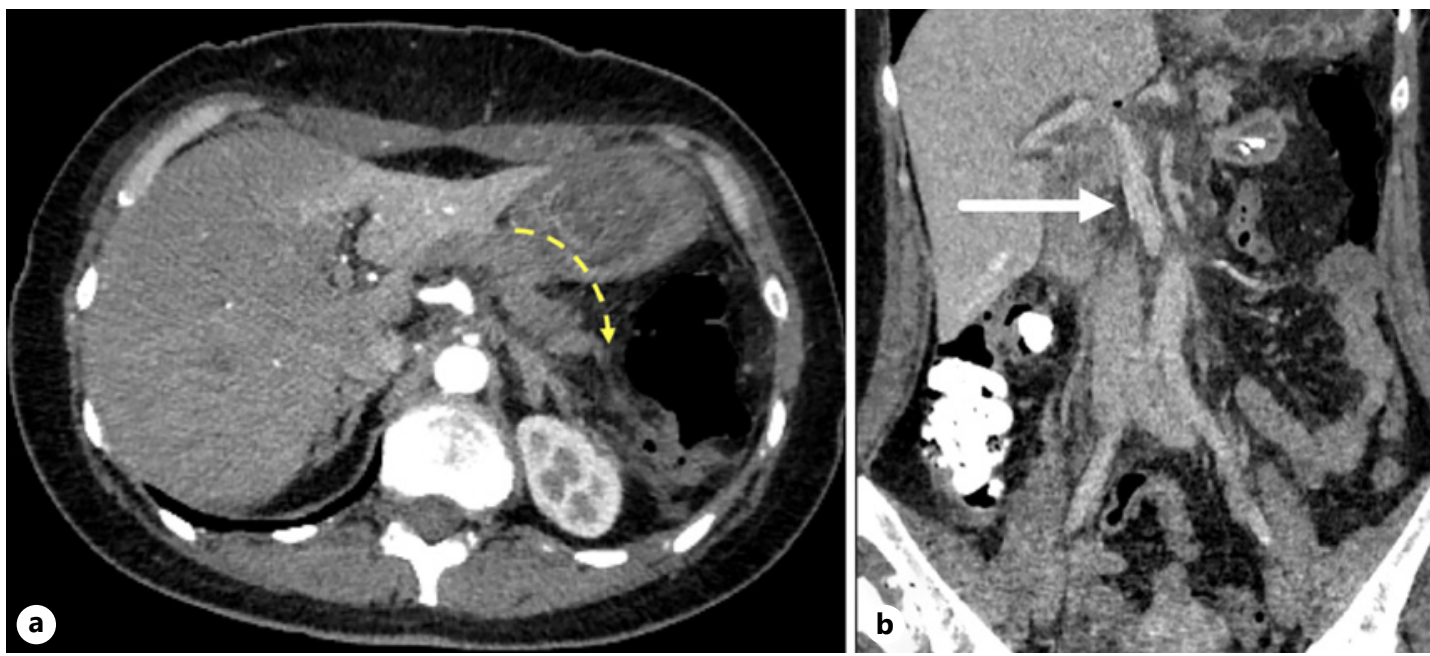

Fig. 3. Postoperative data. a CT scan, native phase, axial view. The dotted line marks the remaining pancreatic parenchyma after surgery. b CT scan, venous phase, frontal view. The arrow indicates a functioning venous prosthesis.

The postoperative period was uneventful. There were no episodes of hyperglycemia. Control CT and ultrasound of the abdominal cavity showed that venous prosthesis functions were adequate, and residual pancreatic parenchyma was without signs of reactive inflammation (Fig. 3). The patient was discharged on the 12th day after surgery in a satisfactory condition for further outpatient control.

Histopathological examination, according to international TNM staging of carcinomas of the pancreas, revealed primary multiple tumors of the pancreas:

1. IPMN associated with invasive well-differentiated ductal adenocarcinoma with invasion to the proximal part of the body, the neck, and partially the upper parts of the head of the pancreas, with lymphovascular and perineural invasion that spread to the peripancreatic adipose tissue and with growth to the portal vein wall in the confluence area (Fig. 2). The maximum size of the tumor was $4.7 \mathrm{~cm}$. There was tumor 
Kriger et al.: Surgical Treatment of Synchronic IPMN and SPN of the Pancreas

spread to adipose tissue of the hepatoduodenal ligament and to the area of portal vein confluence with invasion of the adventitia layer of the wall, as well as growth in 1 lymph node of peripancreatic adipose tissue. The remaining 39 lymph nodes were without signs of metastasis: pT3 pN1 (1/40) cM0; M8453/3; Pn1; L1, V0, R0 (according to the WHO classification of tumors, 5th edition [4]).

2. SPN of the tail of the pancreas, sized $6 \times 5 \times 5.5 \mathrm{~cm}$ with the involvement of peripancreatic adipose tissue: PT3 PN0 (0/40) CM0; M8452/3; low-grade; Pn0; L0; V0; R0. Immunohistochemical study of pancreatic tail tumor: CD99 +; $\beta$-catenin +; CD10 +; CD56 +, vimentin +, chromogranin A -; tripsin (anti) (Cell Marque) -; Ki67 - 4\%, grade 2 (according to the WHO classification of tumors, 5th edition [4]).

The patient subsequently received adjuvant chemotherapy with gemcitabine. The follow-up period was 8 months with no signs of disease recurrence. Further patient observation will be continued.

\section{Discussion}

IPMN of the pancreas was first described by Ohashi and Maruyama [5] in 1982 and is still an uncommon cystic tumor of the pancreas. It occurs in 0.31-4.35 per 100,000 cases per year [1]. The malignant IPMN or so-called IPMC, the malignant form of this tumor, occurs much more rarely. SPN is an extremely rare tumor which was first described in 1959 by the American pathologist Virginia Frantz [2]. It mostly occurs in young females and has progesterone receptors. When we started to exam this female patient, we found 2 neoplasms of the pancreas which were located in the head and the tail of the pancreas. The main sign of malignancy of the pancreatic head tumor was the dilation of the pancreatic duct with its maximal diameter $6 \mathrm{~mm}$. In addition, there seemed to be invasion of the pancreatic head tumor into the mesentericoportal trunk. Therefore, we assumed that the neoplasm of the pancreatic head was malignant.

It was clear that this patient needed to be treated surgically. We dismissed neoadjuvant chemotherapy because we did not know how each of the tumors would respond to the chemotherapy and were afraid of the progressive growth of one of the tumors. We had 3 options: (1) total pancreatoduodenectomy with splenectomy; (2) two-step surgery with first step of pancreaticoduodenectomy; and (3) one-step surgery: pancreaticoduodenectomy with distal pancreatectomy. The first option of total pancreatectomy seemed the easiest to perform and feasible but extremely aggressive, with severe endocrine and exocrine insufficiency in postoperative recovery and during her further life. The second option was quite difficult for us to decide because we did not want to leave such a big tumor in the pancreatic tail without understanding when the second step would be performed. Therefore, for the first time in our practice we decided to use the third method of surgery: the one-step surgery - pancreatoduodenectomy with mesentericoportal trunk resection and distal pancreatectomy, and splenectomy at the same time. During this surgery both tumors could be removed; however, part of the pancreatic tissue could be saved to prevent exocrine and endocrine insufficiency in the long-term follow-up. At the same time, we clearly realized that such a complex operation, which damages the pancreatic parenchyma from both sides, can generate acute postoperative pancreatitis with severe complications such as postpancreatectomy hemorrhage, pancreatic fistula, and even necrosis of pancreatic tissue.

Fortunately for patient and for us, the postoperative recovery was uneventful. We think this was because the pancreatic tissue was adequately atrophic and firm with the dilated pancreatic duct. The success of the surgical volume chosen was evident in the absence of hyperglycemia and exocrine insufficiency after the procedure. The pancreatic tissue remnant provides sufficient hormones and ferments for the normal social activity of the patient. This is quite important for such a young woman. Now the long-term follow-up continues, and the patient receives chemotherapy. 
During the literature search we found only 2 described cases of synchronous IPMC and SPN neoplasms $[6,7]$. In both the described patients, the tumors were located in the same part of the pancreas (pancreatic head and neck). Therefore, doctors performed the typical surgeries and there was no such difficult choice of treatment as in our case. The unusual surgical volume is an extra interesting point of this case.

\section{Conclusion}

We present a case of synchronous SPN with IPMC. The tumors were located in different parts of the pancreas which made the situation much more difficult for surgical treatment. The patient was successfully treated by simultaneous pancreaticoduodenectomy with mesentericoportal trunk resection and distal pancreatectomy, and splenectomy. Oncological and gastroenterological outcomes were satisfactory. There was no exocrine or endocrine insufficiency after surgery.

\section{Statement of Ethics}

Written informed consent to publish their case (including publication of images) was obtained from all individual participants included in the study.

\section{Disclosure Statement}

The authors have no conflicts of interest to declare.

\section{Funding Sources}

This research did not have any funding.

\section{Author Contributions}

A.G.K. and S.V.B. conceived and designed the study, performed the analysis, and wrote the paper. D.S.G., A.R.K., and V.I.P. conceived and designed the study, collected the data, contributed the data, performed the analysis, and wrote the paper. All authors approved the final manuscript.

\section{References}

1 Cortegoso Valdivia P, Chialà C, Venezia L, Gaiani F, Leandro G, Di Mario F, et al. Diagnosis and management of intraductal papillary mucinous neoplasms of the pancreas. Acta Biomed. 2018 Dec;89 (9-S):147-52.

2 Frantz VK. Tumors of the pancreas. In: Bumberg CW, editor. Atlas of tumor pathology. VII. Fascicles 27 and 28. Washington: Armed Forced Institute of Pathology; 1959. pp. 32-9.

3 Kriger A, Kaldarov A, Gorin D, Berelavichus S. Surgical Approach for Solid Pseudopapillary Neoplasm of Pancreas Single - Centre Experience. JOP. J Pancreas (Online). 2018 Mar;19(2):70-4.

4 IARC/WHO. WHO classification of tumors, 5th edition. Available from: https://whobluebooks.iarc.fr/.

5 Ohashi KM, Maruyama M. Four cases of mucin producing cancer of the pancreas on specific findings of the papilla of Vater. Prog Dig Endosc. 1982;20:348-51.

6 Imamura N, Chijiiwa K, Ohuchida J, Hiyoshi M, Takahashi N, Yorita K, et al. Synchronous solid pseudopapillary neoplasm and intraductal papillary mucinous neoplasm of the pancreas: report of a case. Surg Today. 2011 Jun;41(6):865-71.

7 Hirabayashi K, Zamboni G, Ito H, Ogawa M, Kawaguchi Y, Yamashita T, et al. Synchronous pancreatic solid pseudopapillary neoplasm and intraductal papillary mucinous neoplasm. World J Gastroenterol. 2013 Jun; 19(21):3358-63. 